07.2

\title{
Влияние числа рядов GalnAs-квантовых объектов на ток насыщения GaAs-фотопреобразователей
}

\author{
(c) М.А. Минтаиров ${ }^{1}$, В.В. Евстропов ${ }^{2}$, С.А. Минтаиров ${ }^{2}$, А.М. Надточий ${ }^{1}$, Р.А. Салий ${ }^{1}$, М.3. Шварц ${ }^{2}$, \\ Н.A. Калюжный ${ }^{2}$ \\ ${ }^{1}$ НТЦ микроэлектроники РАН, Санкт-Петербург, Россия \\ ${ }^{2}$ Физико-технический институт им. А.Ф. Иофрфе РАН, Санкт-Петербург, Россия \\ E-mail: mamint@mail.ioffe.ru
}

Поступило в Редакцию 12 марта 2020 г.

В окончательной редакции 12 марта 2020 г.

Принято к публикации 24 марта 2020 г.

Исследованы спектры электролюминесценции и зависимости напряжения холостого хода от фотогенерированного тока для GaAs-солнечных элементов, $p-n$-переход которых содержал различное количество рядов $(r)$ квантовых объектов на основе слоев $\mathrm{In}_{0.4} \mathrm{Ga}_{0.6} \mathrm{As}$. Для всех образцов получены значения тока насыщения $\left(J_{0}\right)$, ширины запрещенной зоны квантового объекта $\left(E_{g}^{Q}\right)$ и падения напряжения холостого хода $\left(\Delta V_{o c}\right)$ относительно реферного $(r=0)$ образца. Предложена модель, адекватно описывающая зависимости $J_{0}(r)$ и $\Delta V_{o c}(r)$, и найдены модельные параметры, в том числе токовый инвариант $J_{z}=1.4 \cdot 10^{5} \mathrm{~A} / \mathrm{cm}^{2}$, однозначно связывающий ток насыщения с шириной запрещенной зоны квантового объекта.

Ключевые слова: квантовые объекты, солнечные элементы, фотопреобразователи, ток насыщения.

DOI: 10.21883/PJTF.2020.12.49524.18284

Использование квантовых объектов (квантовых точек, квантовых ям и гибридных объектов) в субэлементах многопереходных солнечных элементов является перспективным методом решения проблемы дисбаланса фотогенерируемых токов субэлементов. Это достигается за счет того, что включенные в субэлемент квантовые объекты (КО) поглощают дополнительные фотоны и, как следствие, позволяют увеличить его фотогенерированный ток. Однако включение КО всегда ведет к понижению напряжения холостого хода субэлемента. Это наблюдается для всех видов квантовых объектов: для квантовых точек [1-3], квантовых ям [4-6] и других объектов (проволочных [7] и гибридных [8-10]). Основной причиной падения напряжения является возникновение дополнительной рекомбинации в КО [3,8-10]. Эти процессы были исследованы нами в $[9,10]$ В частности, в [8,9] была предложена модель, описывающая увеличение тока насыщения $\left(J_{0}\right)$ при введении в $p-n$-переход различного количества рядов $(r)$ КО. Отметим, что ток насыщения напрямую определяет фотонапряжение, генерируемое $p-n$-переходом. Согласно предложенной модели, этот ток линейно увеличивается с $r$, что было подтверждено экспериментально [10] для образцов с количеством рядов КО от 1 до 10. В настоящей работе проведено исследование характеристик $\mathrm{GaAs}$ $p-i-n$-переходов с количеством рядов гибридных КО GaInAs от 1 до 20, а также экспериментально обнаружена и интерпретирована сверхлинейная зависимость $J_{0}$ от $r$. В процессе исследования также было обнаружено, что рекомбинация через уровни в КО не всегда доминирует над рекомбинацией в матрице, что требует совершенствования используемой ранее модели $[9,10]$.
Все исследуемые структуры были выращены методом металлоорганической газофазной эпитаксии, технология выращивания структур описана в [11].

Токи насыщения $J_{0}$ определялись, как и в [8], по анализу экспериментальных характеристик $V_{o c}-J_{g}$ (напряжение холостого хода-фотогенерированный ток), приведенных на рис. 1 (символы). Все характеристики были измерены при достаточно больших фотогенерированных токах $\left(>1 \mathrm{~A} / \mathrm{cm}^{2}\right)$, когда доминирует так называемый диффузионный [12] или единичный [8,9] механизм протекания тока через $p-n$-переход. Единичный ток насыщения определялся путем аппроксимации двухдиодной моделью [8] характеристик $V_{o c}-J_{g}$. Результат аппроксимации показан на рис. 1 (линии), найденные значения единичного тока насыщения приведены в таблице. Полученная зависимость $J_{0}(r)$ была построена. Было определено, что она (при $r>10$ ) сверхлинейна.

Для всех исследуемых образцов были исследованы спектры электролюминесценции (рис. 2), полученные при пропускании через образцы одинакового тока $\left(0.128 \mathrm{~A} / \mathrm{cm}^{2}\right)$. Эти спектры содержали два пика: один от GaAs-матрицы (энергия фотона $1.426 \mathrm{eV}$ интерпретируется как ширина запрещенной зоны $E_{g}$ ), другой от квантовых объектов (от 1.189 до $1.213 \mathrm{eV}$, что характеризует его ширину запрещенной зоны $E_{g}^{Q}$ ). Высота пиков электролюминесценции характеризует темп излучательной рекомбинации через матрицу и КО соответственно. Видно, что при введении одного ряда КО в $p-i-n$-переход высота пика КО незначительно больше, чем высота пика матрицы, что свидетельствует об отсутствии доминирования рекомбинации в КО над рекомбинацией в матрице. С увеличением числа рядов КО рекомбинация 


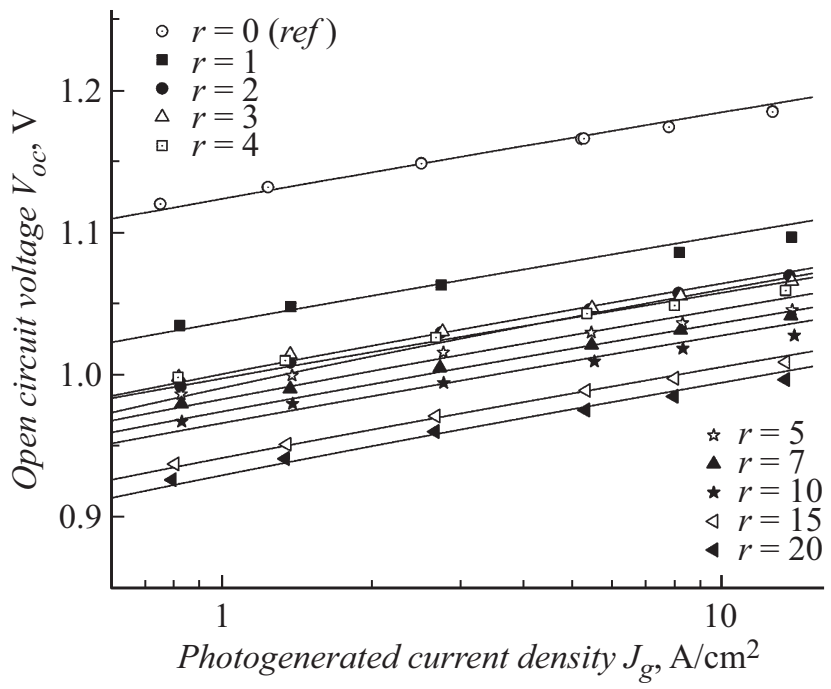

Рис. 1. Зависимости напряжения холостого хода от фотогенерированного тока при разном числе рядов $(r)$ квантовых объектов. Символы - эксперимент, сплошные линии - аппроксимация двухдиодной моделью [8].

Полученные значения параметров исследуемых солнечных элементов

\begin{tabular}{c|c|c|c|c}
\hline $\begin{array}{c}\text { Число рядов КО } \\
r\end{array}$ & $E_{g}, \mathrm{eV}$ & $J_{0}, \mathrm{~A} / \mathrm{cm}^{2}$ & $\Delta E_{g}, \mathrm{eV}$ & $\Delta V_{o c}, \mathrm{~V}$ \\
\hline 0 & 1.426 & $2.5 \cdot 10^{-19}$ & 0.000 & 0.000 \\
(реферный образец) & & & & \\
1 & 1.210 & $9.0 \cdot 10^{-19}$ & 0.2126 & 0.090 \\
2 & 1.202 & $2.9 \cdot 10^{-18}$ & 0.2209 & 0.1189 \\
3 & 1.209 & $3.1 \cdot 10^{-18}$ & 0.2138 & 0.1205 \\
4 & 1.213 & $4.0 \cdot 10^{-18}$ & 0.2091 & 0.1267 \\
5 & 1.203 & $6.0 \cdot 10^{-18}$ & 0.2197 & 0.1370 \\
7 & 1.201 & $9.0 \cdot 10^{-18}$ & 0.2209 & 0.1471 \\
10 & 1.198 & $1.3 \cdot 10^{-17}$ & 0.2243 & 0.1563 \\
15 & 1.195 & $3.0 \cdot 10^{-17}$ & 0.2278 & 0.1772 \\
20 & 1.189 & $4.5 \cdot 10^{-17}$ & 0.2335 & 0.1873
\end{tabular}

в них увеличивается, а при 20 рядах интенсивность пика КО практически на два порядка больше, чем интенсивность пика матрицы. Тем не менее предыдущая модель, описывающая ток насыщения при введении в $p-n$-переход КО, а также величину напряжения, не учитывала тот факт, что рекомбинация через КО не всегда доминирует над рекомбинацией в матрице. Отметим также эффект уменьшения ширины запрещенной зоны КО с увеличением числа рядов КО, что приводит к сверхлинейности зависимости $J_{0}(r)$ (рис. $\left.3, b\right)$. Этот эффект для гибридных КО обсуждался в работе [13], также он наблюдался при включении квантовых ям и квантовых точек [14-16]. Далее нами применена модель, позволяющая корректно описать зависимость тока насыщения и, как следствие, напряжения холостого хода от числа рядов КО (рис. $3, a$ ).
Согласно модели, единичная токовая компонента (с коэффициентом идеальности $A=1$ ) составлена из двух субкомпонент

$$
J=\left(J_{0}^{M}+J_{0}^{Q}\right) \exp \left(\frac{V}{k T / q}\right) .
$$

Одна из них $\left(J_{0}^{M}\right)$ обусловлена рекомбинацией в матрице, другая $\left(J_{0}^{Q}\right)-$ рекомбинацией через набор рядов КО.

Ток насыщения (предэкспонента) матричной субкомпоненты

$$
J_{0}^{M}=J_{z} \exp \left(-\frac{E_{g}}{k T}\right),
$$

где $E_{g}-$ ширина запрещенной зоны матрицы (GaAs), $J_{z}$ - токовый инвариант относительно $E_{g}$ в системе GaInAs $[17,18]$. Ток насыщения КО-субкомпоненты пропорционален числу рядов КО $r$ и также выражается через токовый инвариант

$$
J_{0}^{Q}=\frac{r}{r_{s}} J_{z} \exp \left(-\frac{E_{g}^{Q}}{k T}\right)=\frac{r}{r_{s}} J_{z} \exp \left(-\frac{E_{g}}{k T}\right) \exp \left(\frac{\Delta E_{g}}{k T}\right),
$$

где $r_{s}$ - множитель, учитывающий стремление $\frac{r}{r_{s}} J_{z} \mathrm{~K}$ инварианту $J_{z}$ при увеличении $r$ и его пропорциональность $r$, что удовлетворяется в линейном приближении при $r \ll r_{s}, \Delta E_{g}-$ разница ширин запрещенных зон КО $\left(E_{g}^{Q}\right)$ и матрицы $\left(E_{g}\right)$. Отметим, что выражение (3) является приближенным, в нем множитель $\frac{r}{r_{s}}$ линейно описывает наблюдаемое увеличение темпа рекомбинации через КО с увеличением числа их рядов. Смысл $r_{s}$ заключается в следующем: $r_{s}$ - это такое воображаемое число рядов, при котором набор КО стал бы эквивалентен объемному материалу, имея такое же значение $J_{z}$.

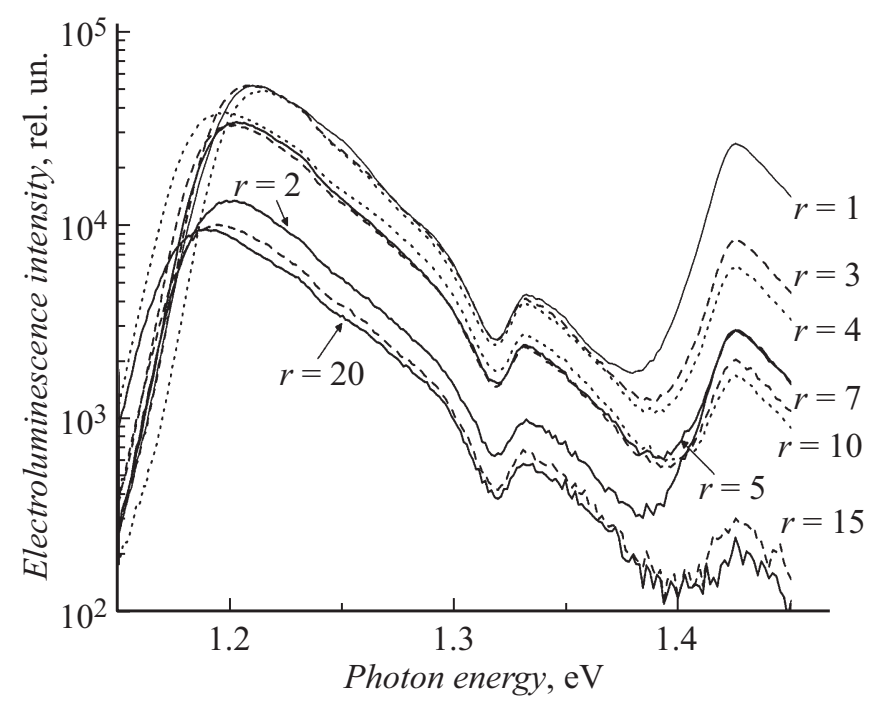

Pис. 2. Спектры электролюминесценции GaAs-солнечного элемента при различном числе рядов $(r)$ квантовых объектов. Провал при $h v \approx 1.1 . \mathrm{eV}$, скорее всего, имеет аппаратурное происхождение. 


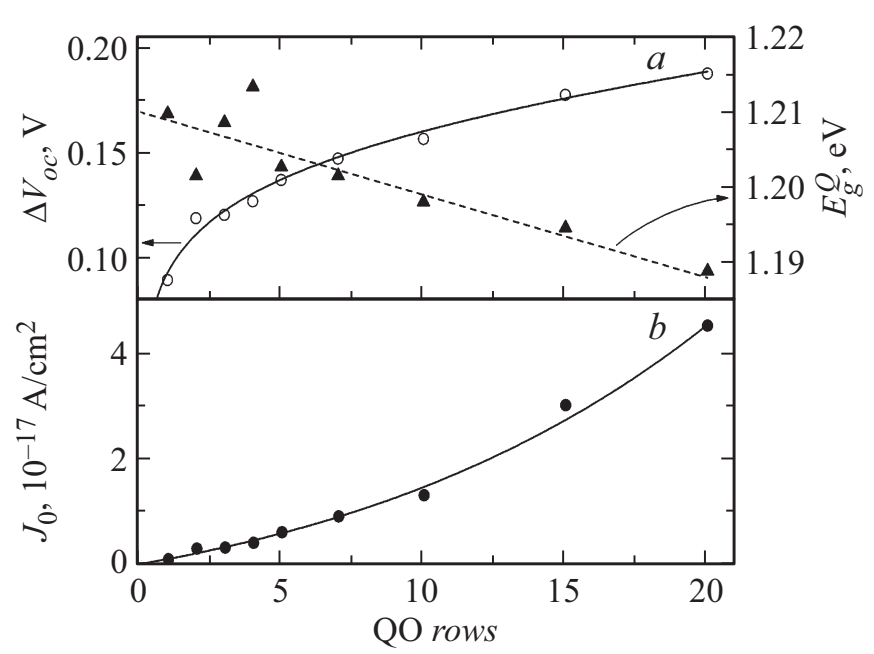

Рис. 3. $a$ - зависимости от числа рядов квантовых объектов падения напряжения холостого хода относительно реферного образца (кружки - эксперимент, сплошная линия - аппроксимация по формуле (7)) и ширины запрещенной зоны квантового объекта (треугольники - эксперимент, штриховая прямая - линейная аппроксимация). $b$ - зависимости от числа рядов квантовых объектов тока насыщения единичной компоненты (кружки - эксперимент, сплошная линия аппроксимация по формуле (4)).

Складывая (2) и (3), получаем суммарный ток насыщения

$$
J_{0}=J_{0}^{M}+J_{0}^{Q}=J_{z} \exp \left(-\frac{E_{g}}{k T}\right)\left[1+\frac{r}{r_{s}} \exp \left(\frac{\Delta E_{g}}{k T}\right)\right] .
$$

Наблюдаемое падение напряжения холостого хода при увеличении числа рядов (рис. 1) моделируется следующим образом. Фотовольтаические характеристики $J_{g}-V_{o c}$ (фотогенерированный ток-напряжение холостого хода) функционально совпадают с темновыми безрезистивными вольт-амперными характеристиками и получаются из них при замене $J \rightarrow J_{g}$ и $V \rightarrow V_{o c}$. Таким образом, фотовольтаические характеристики реферного (без КО, $r=0) p-n$-перехода и изучаемого $(r \geq 1)$ соответственно имеют вид

$$
\begin{gathered}
J_{g}^{M}=J_{0}^{M} \exp \left(-\frac{V_{o c}^{M}}{k T / q}\right), \\
J_{g}^{Q M}=\left(J_{0}^{Q}+J_{0}^{M}\right) \exp \left(-\frac{V_{o c}^{Q M}}{k T / q}\right) .
\end{gathered}
$$

Приравняв $J_{g}^{M}=J_{g}^{Q M}$ и разрешив с учетом (2) и (3) полученное уравнение относительно разницы $V_{o c}^{M}-V_{o c}^{Q M}=\Delta V_{o c}$, находим искомое падение напряжения на участке с $A=1$

$$
\Delta V_{o c}=\frac{k T}{q} \ln \left[1+\frac{r}{r_{s}} \exp \left(\frac{\Delta E_{g}}{k T}\right)\right] .
$$

Предложенная модель была применена к экспериментальным данным. На рис. 3 показаны зависимо- сти от $r$ трех величин: $E_{g}^{Q}, \Delta V_{o c}$ и $J_{0}^{Q}$. Зависимость $E_{g}^{Q}(r)$ была аппроксимирована линейной функцией $E_{g}^{Q}(r)=(1.210-0.011 r) \quad[\mathrm{eV}]$ (рис. 3, $a$, штриховая линия). Функция $E_{g}^{Q}(r)$ позволяет получить $\Delta E_{g}(r)=E_{g}-E_{g}^{Q}(r)$, где значение $E_{g}=1.4255 \mathrm{eV}$ определено экспериментально (рис. 2). С использованием полученной зависимости $\Delta E_{g}(r)$ и выражения (7) была проведена аппроксимация экспериментальной зависимости $\Delta V_{o c}(r)$ (рис. 3, $a$, сплошная линия) и определен безразмерный параметр $r_{s}=140$. Полученные функция $\Delta V_{o c}(r)$ и значение величины $r_{s}$ позволили аппроксимировать при помощи (4) исследуемую зависимость тока насыщения от числа рядов КО (рис. $3, b$, сплошная линия) и определить параметр $J_{z}=1.4 \cdot 10^{5} \mathrm{~A} / \mathrm{cm}^{2}$. Отметим, что этот параметр близок к определенному в работах $[17,18]$, где $J_{z}=2.5 \cdot 10^{5} \mathrm{~A} / \mathrm{cm}^{2}$.

Таким образом, в работе экспериментально определены зависимости $J_{0}, E_{g}^{Q}, \Delta E_{g}$ и $\Delta V_{\text {ос }}$ от числа рядов КО. Обнаружена сверхлинейность зависимости $J_{0}(r)$. Предложена и применена модель, адекватно описывающая исследуемые зависимости, и найдены параметры $r_{s}=140$ и $J_{z}=1.4 \cdot 10^{5} \mathrm{~A} / \mathrm{cm}^{2}$. Модель позволяет описывать ток насыщения $p-n$-перехода с КО, а значит, прогнозировать основные параметры солнечных элементов на его основе, в том числе эффективность.

\section{Благодарности}

Авторы выражают благодарность М.В. Нахимович за измерение спектров электролюминесценции и зависимостей напряжения холостого хода от фотогенерированного тока.

\section{Финансирование работы}

Работа выполнена при поддержке Российского научного фонда (соглашение 17-72-20146).

\section{Конфликт интересов}

Авторы заявляют, что у них нет конфликта интересов.

\section{Список литературы}

[1] Bailey C., Hubbard S., Polly S., Forbes D.V., Raffaelle R.P. // Mater. Res. Soc. Symp. Proc. 2009. V. 1121. P. 1121-N10-02. DOI: https://doi.org/10.1557/PROC-1121-N10-02

[2] Bailey C.G., Forbes D.V., Raffaelle R.P., Hubbard S.M. // Appl. Phys. Lett. 2011. V. 98. P. 163105.

[3] Chan S., Kim D., Sanchez A.M., Zhang Y., Tang M., Wu J., Liu H. // IET Optoelectron. 2019. V. 13. P. 215-217.

[4] Fujii H., Toprasertpong K., Wang Y., Watanabe K., Sugiyama M., Nakano Y. // Prog. Photovolt.: Res. Appl. 2014. V. 22. P. 784-795.

[5] Toprasertpong K., Fujii H., Thomas T., Führer M., Alonso-Álvarez D., Farrell D.J., Watanabe K., Okada Y., Ekins-Daukes N.J., Sugiyama M., Nakano Y. // Prog. Photovolt.: Res. Appl. 2016. V. 24. P. 533-542. 
[6] Ekins-Daukes N.J., Barnes J.M., Barnham K.W.J., Connolly J.P., Mazzer M., Clark J.C., Grey R., Hill G., Pate M.A., Roberts J.S. // Solar Energy Mater. Solar Cells. 2001. V. 68. P. $71-87$.

[7] Sugiyama M., Fujii H., Katoh T., Toprasertpong K., Sodabanlu H., Watanabe K., Alonso-Álvarez D., Ekins-Daukes N.J., Nakano Y. // Prog. Photovolt.: Res. Appl. 2016. V. 24. P. 1606-1614.

[8] Mintairov M.A., Evstropov V.V., Shvarts M.Z., Mintairov S.A., Salii R.A., Kalyuzhnyy N.A. // AIP Conf. Proc. 2016. V. 1748. P. 050003.

[9] Mintairov M.A., Evstropov V.V., Mintairov S.A., Shvarts M.Z., Kalyuzhnyy N.A. // Appl. Phys. Express. 2019. V. 12. P. 035005.

[10] Минтаиров М.A., Евстропов В.В., Минтаиров С.А., Салий Р.А., Швари, М.З., Калюжный Н.А. // ФТП. 2018. T. 52. B. 10. C. $1126-1130$.

DOI: 10.21883/ FTP.2018.10.46451.8878 [Пер. версия: 10.1134/S1063782618100135].

[11] Mintairov S.A., Kalyuzhnyy N.A., Lantratov V.M., Maximov M.V., Nadtochiy A.M., Rouvimov S., Zhukov A.E. // Nanotechnology. 2015. V. 26. P. 385202.

[12] Андреев В.М., Грилихес В.А., Румянцев В.Д. Фотоэлектрическое преобразование концентрированного солнечного излучения. Л.: Наука, 1989. 310 с. [Andreev V.M., Grilikhes V.A., Rumyantsev V.D. Photovoltaic conversion of concentrated sunlight. John Wiley\&Sons Ltd, 1997. 308 p.]

[13] Mintairov S.A., Evstropov V.V., Kalyuzhnyy N.A., Maximov M.V., Mintairov M.A., Nadtochiy A.M., Pavlov N.V., Shvarts M.Z., Zhukov A.E. // Appl. Phys. Express. 2020. V. 13. P. 015009.

[14] Bailey C.G., Forbes D.V., Polly S.J., Bittner Z.S., Dai Y., MacKos C., Raffaelle R.P., Hubbard S.M. // IEEE J. Photovolt. 2012. V. 2. P. 269-275.

[15] Forbes D.V., Hubbard S.M., Bailey C., Polly S., Andersen J., Raffaelle R. // Proc. SPIE. 2010. V. 7772. P. 77720C-1.

[16] Bushnell D.B., Tibbits T.N.D., Barnham K.W.J., Connolly J.P., Mazzer M., Ekins-Daukes N.J., Roberts J.S., Hill G., Airey R. // J. Appl. Phys. 2005. V. 97. P. 124908.

[17] Минтаиров М.A., Евстропов В.В., Минтаиров С.А., Швари, М.З., Калюжный Н.А. // Письма в ЖТФ. 2020. T. 46. B. 7. C. $29-31$.

[18] Mintairov M.A., Evstropov V.V., Mintairov S.A., Shvarts M.Z., Kalyuzhnyy N.A. // J. Phys.: Conf. Ser. 2019. V. 1410. P. 012097. 